\title{
High Q-Factor Wideband Resonators for Millimeter and Submillimeter Applications
}

\author{
Tatiana Gaevskaya, Victor Karpovich, and Valentina Rodionova \\ Institute for Nuclear Problems, Belarus State University, 11 Bobruiskaya Strasse, Minsk 220030, Belarus \\ Correspondence should be addressed to Valentina Rodionova, rodionova@inp.bsu.by \\ Received 8 April 2011; Accepted 22 August 2011 \\ Academic Editor: Bing Liu
}

Copyright ( $) 2012$ Tatiana Gaevskaya et al. This is an open access article distributed under the Creative Commons Attribution License, which permits unrestricted use, distribution, and reproduction in any medium, provided the original work is properly cited.

Physical principles for designing a multipurpose set of high Q-factor quasioptical and corrugated resonators with automatic frequency tuning $\left(Q>6 \times 10^{4}\right.$, VSWR $\left.<1.6\right)$ that can operate in the frequency band from 37.5 to $400 \mathrm{GHz}$ are presented. The electrodynamical calculation methods of resonators, the constructive particularities of resonators, the methods and results of the experimental researches are considered. This set of resonators can be used as a universal measuring resonator for measuring radiosignal fluctuations and parameters of different media, in particular, nanotube composites and high-temperature superconductors.

\section{Introduction}

In recent years, the present-day world of science and practical engineering has seen the UHF- and SHF-band MWtechnology evolving at a vigorous pace. At the same time, research and development work in $\mathrm{mm}$ - and submm-wave technology is still ongoing and gaining momentum; this is mainly because radar, radio navigation, communication equipment, guidance, and control systems tend to enhance their capabilities which can be achieved over these bands alone (specifically, we have to deal with resolving power, detection, hitting of a target, noise immunity, and speed of response) [1-3]. As regards the MW-technology, we are concerned with designing the devices and the components having required parameters. In addition, a series of MWcomponents such as resonators, detectors, mixers, oscillators, attenuators, directional couplers, and others have been engineered not only for the UHF- and MW-band (from 1 to $180 \mathrm{GHz}$ ) but also for submm-band wavelengths (from 180 to $405 \mathrm{GHz}$ ).

The basic advantages of these components are as follows: a broadbandedness, a low losses of power, and a small level of noise. The measuring systems enabling the amplitude and phase characteristics of communication and radar facilities to be determined over the frequency ranges of 1 to $180 \mathrm{GHz}$ are likewise underdevelopment. The noise amplitude measurement is based upon the low-noise detectors for an output power of $1 \mu \mathrm{W}$. The phase noise is measured by means of a two-channel frequency discriminator at an output power of $1 \mathrm{~mW}$. The basic technical characteristics are as follows: (1) the frequency range varies between 1 and $118 \mathrm{GHz}$ (9 models); (2) the measurement sensitivity at a tune-away frequency from a $10 \mathrm{kHz}$ carrier is equal to between $-150 \mathrm{~dB} / \mathrm{Hz}$ and $-165 \mathrm{~dB} / \mathrm{Hz}$ depending upon a model during the measurement of amplitude noise levels; between $-110 \mathrm{~dB} / \mathrm{Hz}$ and $-145 \mathrm{~dB} / \mathrm{Hz}$ during the phase noise measurement.

The systems intended to measure the parameters of different dielectric materials are currently well underdevelopment. A good deal of effort is being undertaken to create new types of transmission lines whose operation is based on the novel physical principles.

High-quality VHF resonators are used to create highquality signal sources of radar and navigation systems, for carrying out spectral and frequency measuring and physical research. VHF resonators are used in VHF electronics: resonance and stabilizing systems of generators, in measuring techniques: the wavemeters, the filters, the measuring instrument for signal spectrum, the frequency discriminators, in experimental physics: a spectroscopy of electronic 
paramagnetic resonance, a measuring of materials parameters, and so forth.

\section{Principles of $Q$-Factor Increasing for mm and Submm Resonators}

At present, the technological possibilities of $Q$-factor increasing under room temperature (use of high-conductivity materials, high-quality processing to surfaces) practically are exhausted. In this context, using new physical principles gains a special role. The simplest approach here: use the higher modes in multimode resonators. However in this case it is necessary to take the supplementary means of the modes selection because of the existence of the degenerate modes in the multimode wideband resonance system. Use of quasioptical opened resonators [4], which are one subject of the present report, is a high effective mean for mode selection. It follows to note that even in them the degree of selection sometimes is insufficient, and it is necessary to use the additional means (see below). In this paper two types of open resonators are considered: type A-with two identical spherical mirrors and type $\mathrm{B}$ - -having a spherical mirror and a planar disk mirror.

The other approach is concluded in using the effect of the reduction of the losses in corrugated surface. This effect was used for reduction of the $\mathrm{H}_{11}$-mode attenuation in flexible cylindrical waveguides [5] and horn antennas [6]. In a given work, the cylindrical resonator with longitudalcorrugated lateral surface (the worker of the mode $E_{\text {omn }}$ with $m=1, \ldots, 2$ and $n=15, \ldots, 25)$ was designed on his base. The method of impedance-type efficient border conditions generalized on event of the final conductivity of the material [6] is a base of electrodynamical calculation of this resonator (Figure 1).

\section{The Wide-Range Matching of Multimode Resonators with Single Mode Rectangular Waveguides}

The simplest coupling element is a diaphragm with round hole ( $R$ radius), located in cross section of waveguide $(R \ll$ $\lambda)$. The calculation of such element is usually conducted on base dipole approach. However, using the usual expression for magnetic polarizability of hole does not lead in this instance to satisfactory consent with experiment. The reason of that is the magnetic polarizability being greatly changed under the action of walls of waveguide [7]. This influence is taken into account in a given work that brings about consent of the theories and experiment.

The nondiaphragm coupling element representing a narrow slot in the center of the spherical mirror is more efficient. It linked a smooth waveguide transition from the slot cross section to that of standard rectangular waveguide, which is orthogonal to the mirrors external surface. The achievement of a high degree of matching in broad range of the frequencies is reached by optimum choice of the irregularity low. The Oliners model, traditionally used for the analysis of microwave integrated circuits, is applied to

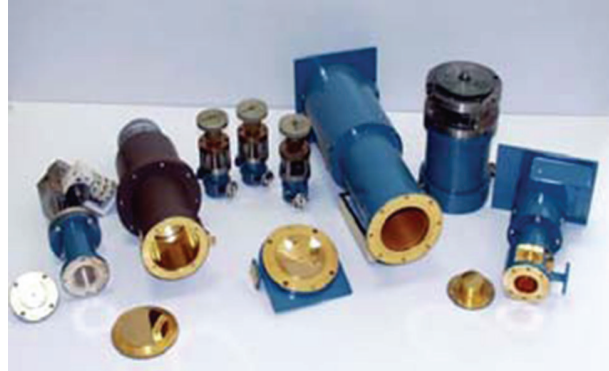

Figure 1: The high Q-factor corrugated and quasioptical resonators.

calculation of the given coupling element. Using the Oliners model, we first pass from the real coupling element to its prototype, which instead of the narrow "electric" walls of the waveguide has "magnetic" ones. The condition of the physical equivalence of the prototype to the given element consists in the equality of the waveguide impedances of operational modes at corresponding cross-sections of the original waveguides and its prototype. For this, the size of the wide wall of the waveguide in the prototype should be $b_{\text {eff }}=b v$, where $v=\left[1-(\lambda / 2 h)^{2}\right]^{1 / 2}$. The operational $\mathrm{TEM}_{\text {onq }}$ oscillation in the resonator will not change its field structure if the "magnetic" walls are continued into the resonator. This Oliners model enables us to easily calculate the power radiated into the waveguide. For decision of the $2 \mathrm{D}$-prototype boundary value problem is used by Galerkins incomplete method with a semi-inversion of the singular operators in the boundary conditions [6].

One more type of the coupling element, investigated in the given work, presents itself as the semitransparent grating of strips applied on a dielectric substrate. This type of coupling element is manufactured involving integral-circuit technology.

\section{Additional Activity for Selection of the Modes}

As is well known, the main principle to selection of the modes in opened resonator is founded on powerfully differing radiation $Q$ factor of the different modes [4]. The additional activity is a use of dissipative selection of the modes. The example considered in the given work, is semitransparent gratings of strips, located above mirror of the resonator (the effect of the resonance absorption in these gratings is used) [8]. In corrugated resonator, the dissipative selection of the modes is realized automatically_ an effect of the anomalous small absorption is very sensitive to corner of the fall, frequency, and polarizations of the field [6].

\section{Experimental Results}

The typical characteristics of the under study resonator are specified in Table 1. All resonators are provided with varactor section for electronic tuning of the frequency. The measured values of VSWR, the passband, and the bandwidth of the electronic tuning of the frequency are presented in Figure 2 
TABLE 1: Typical technical characteristics of resonators.

\begin{tabular}{lcccc}
\hline $\begin{array}{l}\text { Frequency } \\
\text { range, GHz }\end{array}$ & $\begin{array}{c}\text { Type of } \\
\text { resonator }\end{array}$ & $\begin{array}{c}\text { Type of coupling } \\
\text { element }\end{array}$ & Q-factor & VSWR \\
\hline $32,0-37,5$ & & & $6 \times 10^{4}$ & 1,65 \\
$37,5-47,0$ & Corrugated & Wave-guide & $6 \times 10^{4}$ & 1,65 \\
$47,0-53,57$ & & & $6 \times 10^{4}$ & 1,6 \\
\hline $32,0-53,57$ & & & $6 \times 10^{4}$ & 1,65 \\
$53,57-78,33$ & Opened & & $66 \times 10^{4}$ & 1,70 \\
$78,33-118,1$ & quasioptical & Wave-guide & $75 \times 10^{4}$ & 1,70 \\
$118,1-178,4$ & (type A or & & $75 \times 10^{4}$ & 1,90 \\
$178,4-258,0$ & B) & & $85 \times 10^{4}$ & 1,85 \\
$258,0-400,1$ & & & $85 \times 10^{4}$ & 1,85 \\
\hline
\end{tabular}

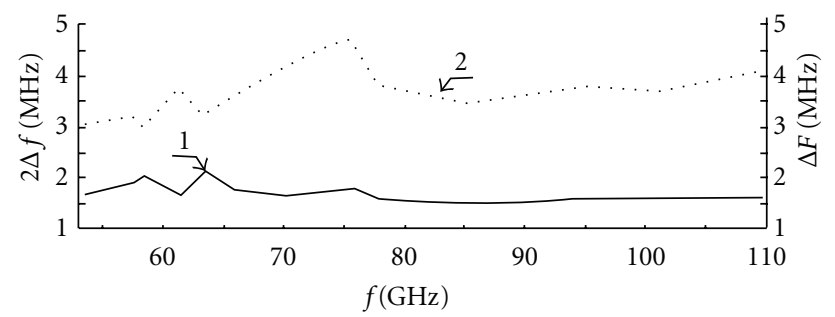

(a)

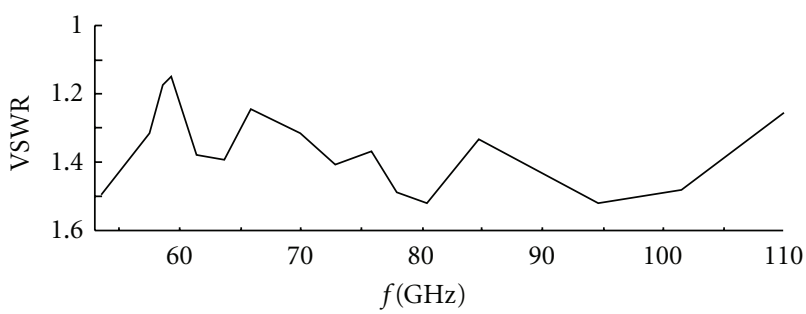

(b)

FIGURE 2: The measured values of VSWR, $2 \Delta f, \Delta F$ as a function of resonant frequency $f$.

as functions of frequency $f$. It can be seen that the varactor section allowed us to obtain the value of automatic electronic frequency tuning of $1 \mathrm{GHz} / \mu$ s without affecting significantly the loaded $Q$-factor of the open resonator (the decrease in the loaded $Q$-factor was so small that its value was comparable with the measurement error).

A uniform electronic timing of an open resonator is obtained. The use of the varactor section located on the ridged waveguide eliminates the dead zones; that is, the frequency regions, in which there is no electronic frequency tuning.

\section{Conclusions}

The results obtained in this paper have been used for designing a multipurpose set of six high $Q$-factor quasioptical resonators with automatic frequency tuning $\left(Q>6 \times 10^{4}\right.$, VSWR $<1,6$ ) which can operate in the frequency band 37.5$400 \mathrm{GHz}$. A series of theoretical and experimental studies has been made into the principles of improving $Q$-factor, mode selection, and wide-band matching of all types of the MW resonators with single-mode waveguides.

The proposed resonators (apart from using them as reference measuring resonators) can be employed to stabilize the oscillator frequency, to measure both the dielectric and magnetic parameters of media, the surface impedance of metals and superconductors, and to measure the insignificant mechanical oscillations. These resonators can likewise be used as wave meters and bandpass filters.

We have also fallen back on the examined concepts of matching the multimode resonators and standard waveguides in developing MW devices that are operated on the principle of microwave heatingup technology.

\section{Acknowledgment}

This work was supported by the National Program "Chemical materials" (Grant 1.14).

\section{References}

[1] A. K. Kamal, “Millimeter wave radar," L'Onde Electrique, vol. 69, no. 6, pp. 70-77, 1989.

[2] A. D. Olver, "Millimetrewave systems-past, present and future," IEE Proceedings-Part F, vol. 136, no. 1, pp. 35-52, 1989.

[3] V. P. Shestopalov, Physical Principles of Millimeter and Submillimeter Engineering, vol. 1 of Open Structures, Scientific Opinion, Kiev, Ukraine, 1985.

[4] L. A. Weinshtein, Open Resonarors and Open Waveguides, Golem Press, 1969.

[5] P. J. B. Clarricoats and A. D. Olver, Corrugated Horns for Microwave Antennas, Peter Peregrinus, London, UK, 1984.

[6] A. S. Ilyinsky, G. Y. Slepyan, and A. Y. Slepyan, Propagation, Scattering and Dissipation of Electromagnetic Waves, Peter Peregrinus, London, UK, 1993.

[7] V. N. Rodionova and G. Ya. Slepyan, "Technical Physics," The Russian Journal of Applied Physics, vol. 59, no. 11, p. 7, 1989.

[8] V. N. Rodionova and G. Y. Slepyan, "Radiotekh," Elektron, vol. 34, no. 7, p. 1358, 1989. 

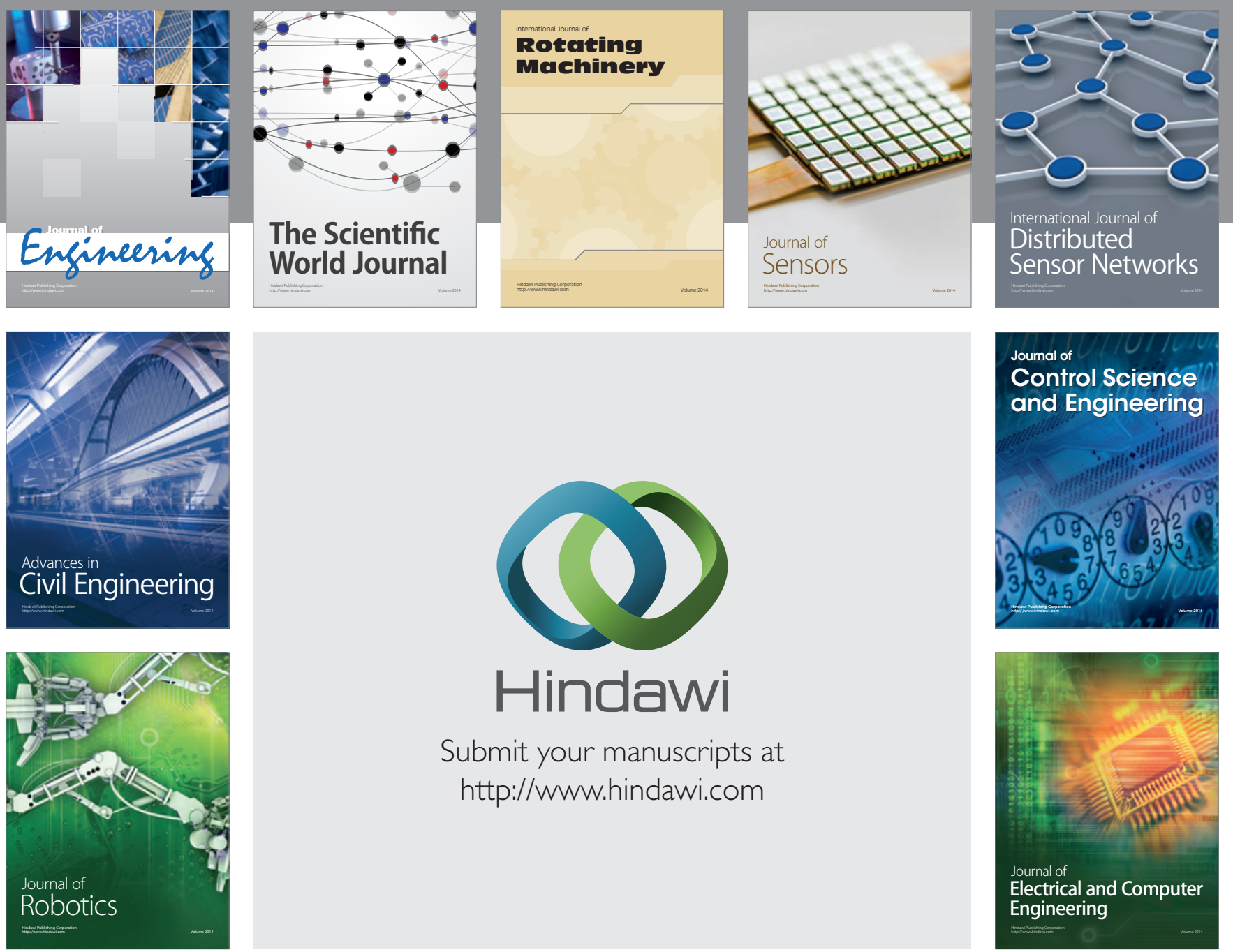

Submit your manuscripts at

http://www.hindawi.com
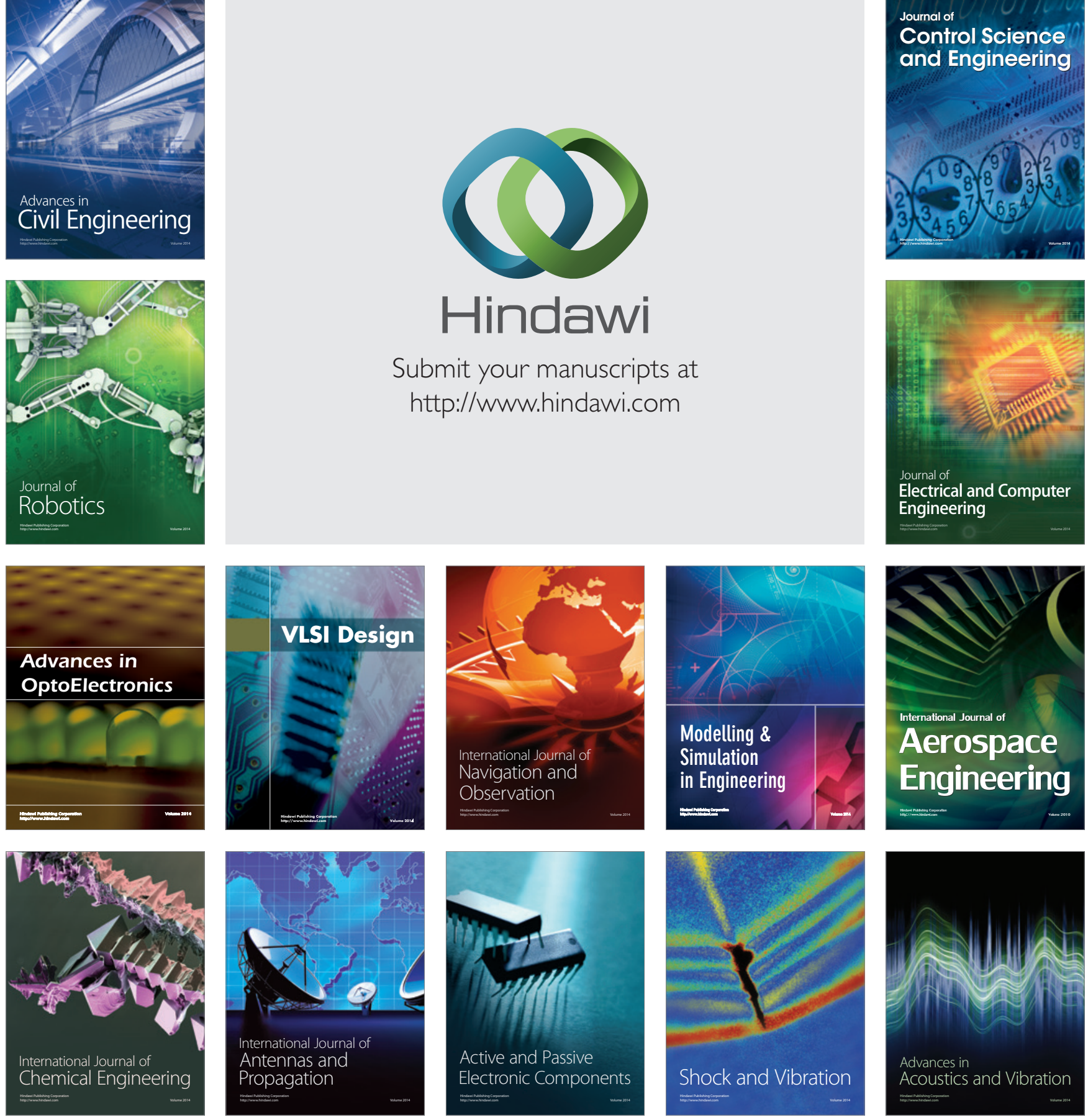